\title{
Magnetoexcitons in phosphorene monolayers, bilayers, and van der Waals heterostructures
}

\author{
Roman Ya. Kezerashvili ${ }^{\circ}$, Anastasia Spiridonova, and Andrew Dublin \\ New York City College of Technology, The City University of New York, Brooklyn, New York 11201, USA \\ and The Graduate School and University Center, The City University of New York, New York, New York 10016, USA
}

(Received 9 August 2021; accepted 9 February 2022; published 24 February 2022)

\begin{abstract}
We study direct and indirect excitons in Rydberg states in phosphorene monolayers, bilayers, and van der Waals (vdW) heterostructure in an external magnetic field within the framework of the effective-mass approximation. The magnetic field is applied perpendicular to the monolayer or heterostructure and is varied between 0 and $60 \mathrm{~T}$. Binding energies of magnetoexcitons are calculated by numerical integration of the Schrödinger equation using the Rytova-Keldysh potential for direct magnetoexcitons and both the Rytova-Keldysh and Coulomb potentials for indirect magnetoexcitons. The latter aids in the understanding of the role of screening in phosphorene. We report the magnetic field energy contribution to the binding energies and diamagnetic coefficients (DMCs) for magnetoexcitons, which depend strongly on the effective anisotropic masses of electrons and holes and can be tuned by the external magnetic field. We demonstrate that the vdW phosphorene heterostructure is a novel category of two-dimensional semiconductors with magnetoexcitonic binding energies tunable by means of the external magnetic field. The binding energies and DMCs are controlled by the number of hexagonal boron nitride layers separating two phosphorene sheets. Such tunability is potentially useful for the device design.
\end{abstract}

DOI: 10.1103/PhysRevResearch.4.013154

\section{INTRODUCTION}

Two-dimensional layered materials have been studied extensively in the past two decades. Initially, the research was focused on graphene (see, for example, [1,2] and references therein) and transitional metal dichalcogenides (TMDCs): $\mathrm{WSe}_{2}, \mathrm{WS}_{2}, \mathrm{MoSe}_{2}$, and $\mathrm{MoS}_{2}$ [3-11]; silicene and other group IV elements followed [12-15] soon after. There are some drawbacks to the above materials. Graphene has high mobility and a large on-off ratio but lacks a gap, impeding its use in the design of electronic devices $[16,17]$. TMDCs have a gap [18-20], but their carrier mobility is orders of magnitude lower than that of graphene. Synthesized in 2014 [21], phosphorene comprising a monolayer of black phosphorus (BP) has advantages over graphene, TMDCs, and Xenes. Its most remarkable properties include strong in-plane anisotropy, a thickness-dependent band gap, and high carrier mobility.

The monolayer of BP appears to be composed of two distinct planes with a puckered honeycomb structure [22-24]. This results in an anisotropic electronic structure. Due to phosphorene's unique topological structure and the differences between the armchair (AC) and zigzag (ZZ) directions, it displays strong in-plane anisotropy. Many properties of phosphorene in these two principal directions are drastically different. The anisotropic structure is strongly reflected in effective masses of charge carriers that lead to anisotropic

Published by the American Physical Society under the terms of the Creative Commons Attribution 4.0 International license. Further distribution of this work must maintain attribution to the author(s) and the published article's title, journal citation, and DOI. effective masses of electrons and holes along the $\mathrm{AC}$ and $\mathrm{ZZ}$ ( $x$ and $y$ ) directions. Along the AC direction, the effective electron and hole masses are smaller than those along the $\mathrm{ZZ}$ direction [22-25]. The distinct feature of BP is that the direct gap located at the $\Gamma$ point is preserved in the monolayer and bulk structure. Therefore, phosphorene is a direct-band-gap semiconductor with a strongly anisotropic dispersion in the vicinity of the gap. It is worth noting that the unusual structure of phosphorene distinguishes it from graphene and other widely studied two-dimensional (2D) semiconductors.

Since its synthesis, phosphorene has been studied extensively. For example, optical and thermal properties have been considered in Refs. [24,26-29]; the in-plane electric field has been studied in Refs. [30-33]; the effects of the strain on different properties of phosphorene have been addressed in Refs. [34-36]; and the Landau levels have been reported in Refs. [37-42].

Phosphorene hosts tightly bound excitons [22,43-46]. As with other monolayer semiconductors, both reduced dimensionality and reduced screening of the Coulomb attraction lead to a high exciton binding energy in phosphorene. Moreover, the exciton binding energy in phosphorene is larger than the binding energy in other 2D materials. Excitons in semiconductors subject to external magnetic fields have been studied for the past 60 years. Elliot and Loudon [47] and Hasegawa and Howard [48] developed the theory of the Mott exciton in a strong magnetic field. The authors of Refs. [49-51] addressed Mott excitons. Excitons in TMDC monolayers, bilayers, and vdW heterostructures in the presence of external magnetic fields have been studied extensively. Diamagnetic shifts have been reported in Refs. [11,52-59], and Zeeman shifts have been reported in Refs. [60-65]. We cite these works, but the recent literature on the subject is not 


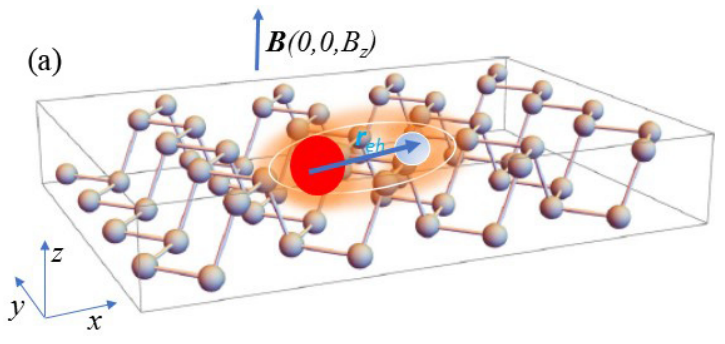

(c)

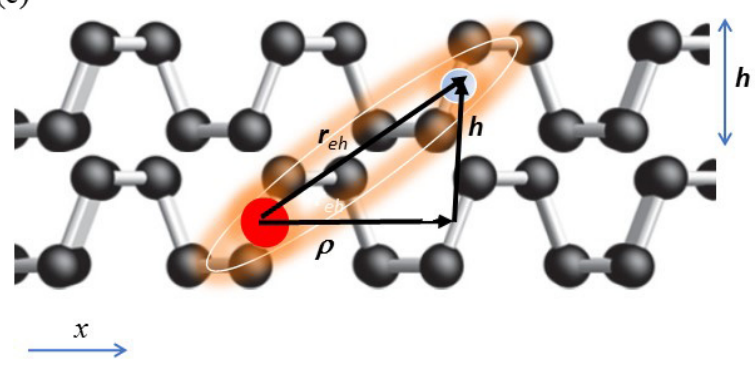

(b)

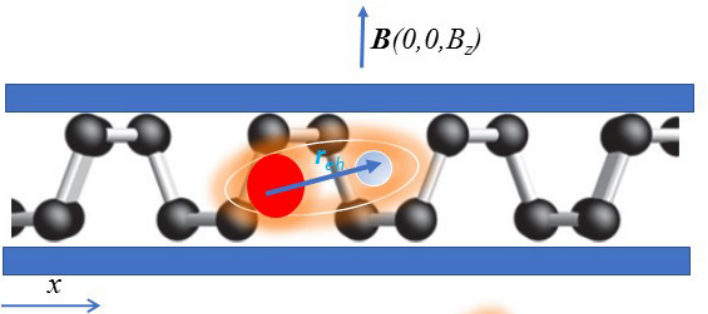

(d)

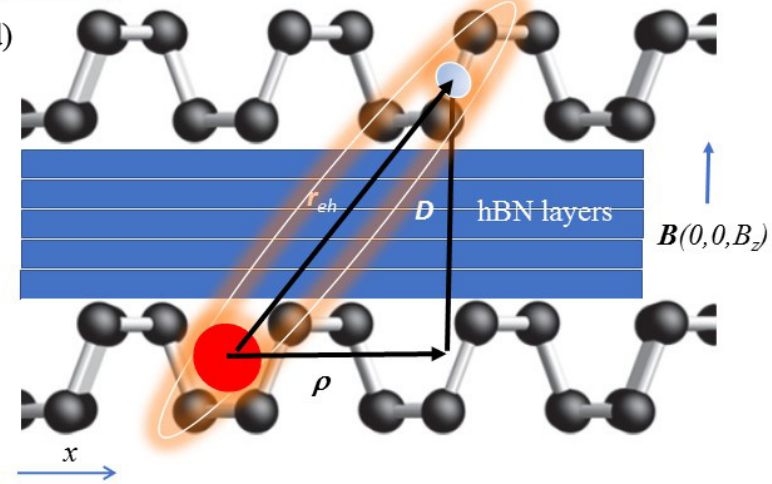

FIG. 1. Schematic illustration of magnetoexcitons in phosphorene monolayers and heterostructures. (a) A direct magnetoexciton in a freestanding phosphorene monolayer. (b) A direct magnetoexciton in an encapsulated phosphorene monolayer. (c) An indirect magnetoexciton in a freestanding bilayer phosphorene. (d) An indirect magnetoexciton in a phosphorene van der Waals heterostructure.

limited by them. There is, however, a lack of similar research on excitons in phosphorene. This motivates us to study the effect of external magnetic fields on the binding energies of Rydberg states of magnetoexcitons in monolayer, bilayer, and $\mathrm{vdW}$ heterostructures composed of phosphorene, and to calculate the corresponding diamagnetic coefficients (DMCs).

In this paper, we study the dependence of the magnetoexciton binding energy of Rydberg states $(1 s, 2 s, 3 s$, and $4 s)$ on the external magnetic field perpendicular to a monolayer or heterostructure. We report the diamagnetic coefficients (i) for the direct magnetoexcitons in freestanding (FS) and encapsulated phosphorene monolayers, (ii) for the indirect magnetoexciton in a bilayer composed of two phosphorene monolayers, and (iii) for the indirect magnetoexcitons in heterostructures formed by two phosphorene monolayers separated by $N$ hexagonal boron nitride (hBN) monolayers. The latter van der Waals heterostructure is denoted as vdW. In our approach, we numerically solve the Schrödinger equation for the magnetoexciton and obtain eigenfunctions and eigenvalues. We then obtain the energy contribution of the magnetic field to the binding energies and use it to calculate diamagnetic coefficients. We solve the Schrödinger equation with the Rytova-Keldysh (RK) potential $[66,67]$ for direct magnetoexcitons and with the RK and Coulomb potentials for indirect magnetoexcitons. The latter allows us to understand the role of screening in phosphorene.

The remainder of this paper is organized as follows: In Sec. II a theoretical model is presented for the description of an electron-hole system in an external magnetic field with charge carrier effective-mass anisotropy. In Sec. III we present the results of calculations of binding energies of Rydberg states and DMCs for direct magnetoexcitons in freestanding and encapsulated phosphorene, as well as the binding energies of indirect magnetoexcitons in FS bilayers and vdW phosphorene heterostructures. Conclusions follow in Sec. IV.

\section{THEORETICAL MODEL}

It is known that electrostatically bound electrons and holes in an external magnetic field form magnetoexcitons. In this section, following Refs. [11,15,50,59], we introduce briefly the theoretical model for the description of the Mott-Wannier magnetoexciton in phosphorene. We consider the energy contribution, $\Delta E$, of the external magnetic field to the Rydberg state binding energies, $E_{B}$, of magnetoexcitons and DMCs. In the system under consideration, excitons are confined to a 2D freestanding and encapsulated by hBN phosphorene monolayer, FS bilayer phosphorene, and van der Waals heterostructure, where $N$ layers of $\mathrm{hBN}$ monolayers separate two phosphorene monolayers. In the latter two cases, an equal number of electrons and holes are separated in parallel phosphorene monolayers by a distance $D$. The corresponding schematic illustrations of these systems are shown in Fig. 1.

It is worth mentioning that we are considering the monolayers of hBN as insulators. Phosphorene encapsulated between hBN layers is sensitive to oxidation in air and exhibits high mobility [68-70]. Moreover, hBN has a high dielectric constant, resulting in a strong damping of the electrostatic repulsion by charged impurities that contribute to a decrease in carrier mobility.

Let us introduce the coordinate vectors of the electron and hole for the Mott-Wannier exciton in the phosphorene layer. The following in-plane coordinates $\mathbf{r}_{e}\left(x_{e}, y_{e}\right)$ and $\mathbf{r}_{h}\left(x_{h}, y_{h}\right)$ for an electron and hole, respectively, are used in our description. We assume that at low momentum $\mathbf{p}\left(p_{x}, p_{y}\right)$, i.e., near the $\Gamma$ point, the single electron and hole energy spectrum $\varepsilon_{l}^{(0)}(\mathbf{p})$ is given by

$$
\varepsilon_{l}^{(0)}(\mathbf{p})=\frac{p_{x}^{2}}{2 m_{x}^{l}}+\frac{p_{y}^{2}}{2 m_{y}^{l}}, \quad l=e, h,
$$


where $m_{x}^{l}$ and $m_{y}^{l}$ are the electron and hole effective masses along the $x$ and $y$ directions, respectively.

The anisotropic nature of phosphorene, unlike other 2D isotropic materials such as graphene and TMDC semiconductors, breaks the central symmetry and requires a description of excitons using Cartesian coordinates. The asymmetry of the electron and hole dispersion in phosphorene is reflected in the Hamiltonian for the Mott-Wannier magnetoexciton; within the framework of the effective-mass approximation, the Hamiltonian for an interacting electron-hole pair in phosphorene in the external magnetic field reads $(\hbar=c=1)$

$$
\begin{aligned}
\hat{H}= & \frac{1}{2 m_{x}^{e}}\left(i \nabla_{x}^{e}-e A_{x}\left(r_{e}\right)\right)^{2}+\frac{1}{2 m_{y}^{e}}\left(i \nabla_{y}^{e}-e A_{y}\left(r_{e}\right)\right)^{2} \\
& +\frac{1}{2 m_{x}^{h}}\left(i \nabla_{x}^{h}+e A_{x}\left(r_{h}\right)\right)^{2}+\frac{1}{2 m_{y}^{h}}\left(i \nabla_{y}^{h}+e A_{y}\left(r_{h}\right)\right)^{2} \\
& +V\left(\left|\mathbf{r}_{e}-\mathbf{r}_{h}\right|\right),
\end{aligned}
$$

where $m_{i}^{j}, i=x, y, j=e, h$ correspond to the effective mass of the electron or hole in the $x$ or $y$ direction, respectively, and $V\left(\left|\mathbf{r}_{e}-\mathbf{r}_{\mathbf{h}}\right|\right)$ describes the electrostatic interaction between the electron and hole. When the electron and hole are both located in a 2D plane, we use the Rytova-Keldysh potential $[66,67]$, which is widely used to describe charge carrier interactions in 2D materials. The RK potential is a central potential, and the interaction between the electron and hole for direct excitons in a monolayer has the form $[66,67]$

$$
V_{\mathrm{RK}}(r)=-\frac{\pi k e^{2}}{2 \kappa \rho_{0}}\left[H_{0}\left(\frac{r}{\rho_{0}}\right)-Y_{0}\left(\frac{r}{\rho_{0}}\right)\right],
$$

where $r=\left|\mathbf{r}_{e}-\mathbf{r}_{\mathbf{h}}\right|$ is the distance between the electron and hole. In Eq. (3), $e$ is the charge of the electron, $\kappa=\left(\epsilon_{1}+\right.$ $\left.\epsilon_{2}\right) / 2$ describes the surrounding dielectric environment, $\epsilon_{1}$ and $\epsilon_{2}$ are the dielectric constants below and above the monolayer, respectively, $H_{0}$ and $Y_{0}$ are the Struve and Bessel functions of the second kind, respectively, and $\rho_{0}$ is the screening length. The screening length $\rho_{0}$ can be written as $\rho_{0}=2 \pi \chi_{2 \mathrm{D}} / \kappa$ [71], where $\chi_{2 \mathrm{D}}$ is the $2 \mathrm{D}$ polarizability, as given in [67]. At long-range distances, $V_{\mathrm{RK}}$ retains the $1 / r$ behavior of the Coulomb potential; for smaller distances, the potential well is logarithmic.

Following the standard procedure [72] for the separation of the relative motion of the electron-hole pair from its centerof-mass motion, one introduces variables for the center of mass of an electron-hole pair, $\mathbf{R}(X, Y)$, and the relative motion, $\mathbf{r}(x, y)$, as $X=\left(m_{x}^{e} x_{e}+m_{x}^{h} x_{h}\right) /\left(m_{x}^{e}+m_{x}^{h}\right), Y=\left(m_{y}^{e} y_{e}+\right.$ $\left.m_{y}^{h} y_{h}\right) /\left(m_{y}^{e}+m_{y}^{h}\right)$, and $x=x_{e}-x_{h}, y=y_{e}-y_{h}, r^{2}=x^{2}+$ $y^{2}$. The Schrödinger equation with Hamiltonian (2) has the form $\hat{H} \Psi\left(\mathbf{r}_{e}, \mathbf{r}_{h}\right)=\mathcal{E} \Psi\left(\mathbf{r}_{e}, \mathbf{r}_{h}\right)$, where $\Psi\left(\mathbf{r}_{e}, \mathbf{r}_{h}\right)$ and $\mathcal{E}$ are the eigenfunction and eigenenergy, respectively. One can write $\Psi\left(\mathbf{r}_{e}, \mathbf{r}_{h}\right)$ in the form $\Psi\left(\mathbf{r}_{e}, \mathbf{r}_{h}\right)=\Psi(\mathbf{R}, \mathbf{r})=e^{i \mathbf{P} \cdot \mathbf{R} / \hbar} \Phi(x, y)$, where $\mathbf{P}=\left(P_{x}, P_{y}\right)$ is the momentum of the center of mass of the electron-hole pair, and $\Phi(x, y)$ is the wave function of the relative motion of the electron-hole pair. Following Refs. [11,15,59,73], after lengthy calculations, one obtains the equation that describes the Mott-Wannier exciton in the Rydberg optical states in an external magnetic field perpendicular to the phosphorene monolayer. The equation for the relative motion of the electron and hole in the phosphorene monolayer
TABLE I. Parameters for phosphorene. Four sets of reduced masses for phosphorene are used in calculations as input parameters. The anisotropic reduced masses $\mu_{x}$ and $\mu_{y}$ are obtained based on effective masses of the electron and hole given in Ref. [75] for set 1, in Ref. [76] for set 2, in Ref. [77] for set 3, and in Ref. [68] for set 4. $\mu_{x}$ and $\mu_{y}$ are in units of the electron mass, $m_{0} \cdot \chi_{2 \mathrm{D}}$ and $h$ are the polarizability and thickness of phosphorene, respectively, in $\mathrm{nm}$.

\begin{tabular}{lccrcc}
\hline \hline & \multicolumn{1}{c}{$\mu_{x}$} & \multicolumn{1}{c}{$\mu_{y}$} & $\mu_{y} / \mu_{x}$ & $\chi_{2 \mathrm{D}}(\mathrm{nm})$ & $h(\mathrm{~nm})$ \\
\hline set 1 & 0.06296 & 0.96774 & 15.37 & $0.41^{\mathrm{a}}$ & $0.541^{\mathrm{b}}$ \\
set 2 & 0.06667 & 0.88780 & 13.32 & & \\
set 3 & 0.09122 & 0.6599 & 7.23 & & \\
set 4 & 0.07969 & 0.9498 & 11.92 & & \\
\hline \hline
\end{tabular}

${ }^{\mathrm{a}}$ Reference [78].

${ }^{\mathrm{b}}$ Reference [79].

with zero center-of-mass momentum $[50,73,74]$ reads

$$
\begin{aligned}
& {\left[-\frac{1}{2 \mu_{x}} \frac{\partial^{2}}{\partial x^{2}}-\frac{1}{2 \mu_{y}} \frac{\partial^{2}}{\partial y^{2}}+\frac{e^{2}}{8 \mu_{x}} B^{2} x^{2}\right.} \\
& \left.+\frac{e^{2}}{8 \mu_{y}} B^{2} y^{2}+V(x, y)\right] \Phi(x, y)=E \Phi(x, y),
\end{aligned}
$$

where $\mu_{x}=\frac{m_{x}^{e} m_{x}^{h}}{m_{x}^{e}+m_{x}^{h}}$ and $\mu_{y}=\frac{m_{y}^{e} m_{y}^{h}}{m_{y}^{e}+m_{y}^{h}}$ are the anisotropic reduced masses in the $x$ and $y$ directions, respectively. In Eq. (4), the anisotropy manifests in the kinetic and magnetic terms, whereas the potential term has an isotropic form.

Let us now consider the indirect magnetoexcitons formed by electrons and holes located in two different phosphorene monolayers in the bilayer or vdW heterostructure. In the latter case, the phosphorene layers are separated by $N$ layers of hBN monolayers. Equation (4) still describes the indirect exciton. However, for indirect excitons, the expression for the interaction between the electron and hole in Eq. (4) is given by

$$
\begin{aligned}
& V_{\mathrm{RK}}\left(\sqrt{\rho^{2}+D^{2}}\right) \\
& =-\frac{\pi k e^{2}}{2 \kappa \rho_{0}}\left[H_{0}\left(\frac{\sqrt{\rho^{2}+D^{2}}}{\rho_{0}}\right)-Y_{0}\left(\frac{\sqrt{\rho^{2}+D^{2}}}{\rho_{0}}\right)\right]
\end{aligned}
$$

for the RK potential, and

$$
V_{C}\left(\sqrt{\rho^{2}+D^{2}}\right)=-\frac{k e^{2}}{\kappa\left(\sqrt{\rho^{2}+D^{2}}\right)}
$$

for the Coulomb potential, where $\rho^{2}=x^{2}+y^{2}$. Equations (5) and (6) describe the interaction between the electron and hole located in different parallel phosphorene monolayers separated by a distance $D=h+N l_{\mathrm{hBN}}$, where $l_{\mathrm{hBN}}=0.333 \mathrm{~nm}$ is the thickness of the hBN layer and $h$ is the phosphorene thickness given in Table I. Therefore, one can obtain the eigenfunctions and eigenenergies of magnetoexcitons by solving Eq. (4) with the potential (3) for direct magnetoexcitons, or by using either potential (5) or (6) for indirect magnetoexcitons. 
(a)

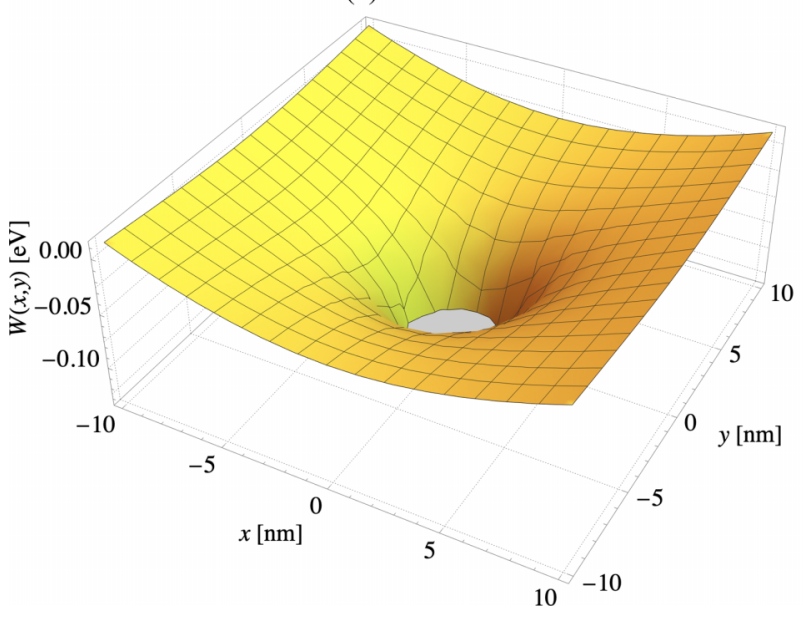

(b)

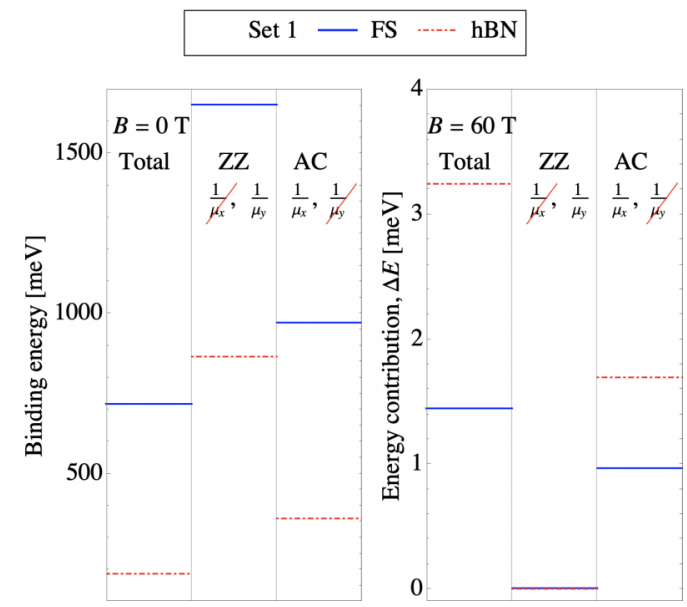

FIG. 2. (a) Total potential $W(x, y)=V_{\mathrm{RK}}(x, y)+\frac{e^{2}}{8 \mu_{x}} B^{2} x^{2}+\frac{e^{2}}{8 \mu_{y}} B^{2} y^{2}$ is plotted as a function of $x$ and $y$. $W(x, y)$ and $x$ and $y$ are given in $\mathrm{eV}$ and $\mathrm{nm}$, respectively. The potential is calculated at $B=30 \mathrm{~T}$ with the parameters of the reduced masses of set 1 . (b) Diagrams of the energy levels of excitons at $B=0 \mathrm{~T}$, and the contribution of the magnetic field at $B=60 \mathrm{~T}$. The crossed-out term means that corresponding terms in Eq. (4) are omitted. Data are given for FS and hBN-encapsulated phosphorene monolayers.

\section{RESULTS OF CALCULATIONS AND DISCUSSION}

We report the dependence of the energy contribution of the external magnetic field on the binding energies of magnetoexcitons in Rydberg states $(1 s, 2 s, 3 s$, and $4 s)$ in FS [Fig. 1(a)] and hBN-encapsulated phosphorene [Fig. 1(b)], the FS bilayer phosphorene [Fig. 1(c)], and the vdW heterostructure [Fig. 1(d)] on a magnetic field. The diamagnetic coefficients for the phosphorene monolayers, the bilayer, and the $\mathrm{vdW}$ heterostructure are reported.

Phosphorene exhibits charge carrier effective-mass anisotropy with lighter effective masses of electron and holes along the armchair direction, and heavier effective masses along the zigzag direction. This leads to the corresponding anisotropic reduced masses $\mu_{x}$ and $\mu_{y}$ in the $\mathrm{AC}$ and $\mathrm{ZZ}$ directions with $\mu_{y}>\mu_{x}$. In our calculations, we use effective masses for the electron and hole from Refs. [68,75-77], which are obtained from first-principles. The lattice constants in Refs. [68,75-77] do not coincide with each other; different functionals for the correlation energy and setting parameters for the hopping lead to some differences in results for anisotropic masses. It is obvious that this may give rise to discrepancies in the band curvatures and, therefore, in the effective masses along the armchair and zigzag directions.

For the set of masses presented in Table I, the ratio $\mu_{y} / \mu_{x}$ varies from $\sim 7$ to 16 . Therefore, from Eq. (4), one can conclude that contributions from the kinetic and magnetic energies to the total ground-state energy are of different orders of magnitude along the $\mathrm{AC}$ and $\mathrm{ZZ}$ directions. In particular, the kinetic energy along the $\mathrm{AC}$ direction is much larger than that along the $\mathrm{ZZ}$ direction. Thus, the anisotropic nature of $2 \mathrm{D}$ phosphorene atomic semiconductors, in contrast to other $2 \mathrm{D}$ materials such as graphene, Xene, and TMDC semiconductors, allows excitons to be confined in a quasi-one-dimensional space, as predicted in theory $[68,80]$, leading to remarkable phenomena arising from reduced dimensionality and screening. Figure 2(a) presents the total potential $W(x, y)=V_{\mathrm{RK}}(x, y)+\frac{e^{2}}{8 \mu_{x}} B^{2} x^{2}+$ $\frac{e^{2}}{8 \mu_{y}} B^{2} y^{2}$ acting on the electron-hole system in phosphorene. One can observe the anisotropic structure of this potential and its asymmetry with respect to the $\mathrm{AC}$ and $\mathrm{ZZ}$ directions.

The effective masses are input parameters in Eq. (4). Different sets of parameters naturally lead to different eigenvalues and eigenfunctions for the Schrödinger equation. This motivates us to utilize the sets of masses given in Refs. [68,75-77] to understand the effect of the anisotropic effective masses of electrons and holes on binding energies and DMCs of magnetoexcitons. The corresponding reduced masses $\mu_{x}$ and $\mu_{y}$ are given in Table I. We refer to these masses as set 1 , set 2 , set 3 , and set 4 . Below, we report results for the sets of masses 1 and 3 . Since sets 1 and 3 give upper and lower bounds on the ratio $\mu_{y} / \mu_{x}$, we can examine systems with higher and lower asymmetry. Furthermore, set 1 has the lowest $\mu_{x}$ and the highest $\mu_{y}$ (and vice versa for set 3).

In studies of excitons in isotropic two-dimensional materials, one obtains the one-dimensional radial equation. The description of excitons in anisotropic materials requires us to solve the two-dimensional Eq. (4). A numerical solution of the Schrödinger equation (4) is performed using the finiteelement method implemented in Wolfram MATHEMATICA's NDEigensystem function, which yields pairs of eigenenergies and eigenfunctions corresponding to the most-strongly-bound states. We modify the code successfully implemented in Ref. [28] in a way that explicitly contains the terms $\frac{e^{2}}{8 \mu_{x}} B^{2} x^{2}$ and $\frac{e^{2}}{8 \mu_{y}} B^{2} y^{2}$. The difference between the codes for the solution of the Schrödinger equation for isotropic [11,15,59] and anisotropic systems is as follows: for isotropic systems we solve the one-dimensional radial Schrödinger equation, and for anisotropic systems we solve the two-dimensional Schrödinger. To check the validity of the code, we use the input parameters from respective papers listed below and calculate the binding energies of direct and indirect excitons. The code reproduces the theoretical binding energies of direct 
TABLE II. Binding energies of direct magnetoexcitons in FS and hBN-encapsulated phosphorene monolayers, as well as indirect magnetoexcitons in FS bilayer phosphorene, for the sets of masses 1 and 3. For indirect magnetoexcitons in bilayer phosphorene, binding energies are calculated using $V_{\mathrm{RK}}$ and $V_{C}$ potentials. Energies are given in $\mathrm{meV}$.

\begin{tabular}{|c|c|c|c|c|c|c|c|c|}
\hline \multirow[b]{3}{*}{ State } & \multicolumn{4}{|c|}{ Monolayer } & \multicolumn{4}{|c|}{ Bilayer } \\
\hline & \multicolumn{2}{|c|}{ Set 1} & \multicolumn{2}{|c|}{ Set 3} & \multicolumn{2}{|c|}{ Set 1} & \multicolumn{2}{|c|}{ Set 3} \\
\hline & $\mathrm{hBN}$ & FS & $\mathrm{hBN}$ & FS & $V_{\mathrm{RK}}$ & $V_{C}$ & $V_{\mathrm{RK}}$ & $V_{C}$ \\
\hline $1 s$ & 187.94 & 718.71 & 199.66 & 746.06 & 388.88 & 827.17 & 395.54 & 854.03 \\
\hline $2 s$ & 78.62 & 488.84 & 74.37 & 478.75 & 298.56 & 653.05 & 294.50 & 644.97 \\
\hline $3 s$ & 53.83 & 394.04 & 50.35 & 377.72 & 248.52 & 525.71 & 239.53 & 501.07 \\
\hline $4 s$ & 35.67 & 319.55 & 32.03 & 311.22 & 211.61 & 430.68 & 209.07 & 429.89 \\
\hline
\end{tabular}

excitons in phosphorene and of indirect excitons in bilayer reported in Ref. [81] within $8 \%$, where binding energies are calculated using Wannier effective-mass theory in 2D space. The binding energies for the $1 s$ state obtained using a semianalytical perturbation theory approach [46] are reproduced within $4 \%$. The code reproduces binding energies for $n=$ $0, \ldots, 4$ states for FS phosphorene, phosphorene on $\mathrm{SiO}_{2}$ substrate, and hBN-encapsulated phosphorene within $1 \%$ when the Schrödinger equation is solved via a finite-element representation of the exciton wave equation [82] and within $4 \%$ when solved using $k \cdot p$ theory [40].

Table II gives our results for binding energies of direct excitons in FS and encapsulated hBN phosphorene monolayers as well as for indirect excitons in FS bilayer phosphorene using the masses in sets 1 and 3. One can see that binding energies of excitons in FS and encapsulated monolayers depend heavily on the anisotropic reduced masses. Moreover, a diminished screening of excitons in the freestanding monolayer gives rise to a threefold increase in the ground-state binding energies relative to those of $\mathrm{hBN}$-encapsulated monolayers.

\section{A. Contribution of the external magnetic field to binding energies of magnetoexcitons in a monolayer}

In Figs. 3(a) and 3(b), we report the energy contribution of the external magnetic field to the binding energies of Rydberg states for direct magnetoexcitons in FS and direct magnetoexcitons in hBN-encapsulated phosphorene as a function of $B^{2}$.

Interestingly enough, for binding energies of excitons and magnetoexcitons in phosphorene, as well as $E_{B}$ of magnetoexcitons in TMDC [11,59] and Xene [15] monolayers, we observe a strong dependence on the reduced mass: $E_{B}$ increases while $\Delta E$ decreases with increased reduced mass. One can say that these dependencies follow directly from Eq. (4), where the mass is one of a few parameters. However, phosphorene demonstrates interesting phenomena due to the mass anisotropy along the $x$ and $y$ directions. On the one hand, higher $\mu_{y}$ leads to a sizable binding energy but a negligible energy contribution of the magnetic term. On the other hand, smaller $\mu_{x}$ gives little contribution to the binding energies but sizable energy contributions from the magnetic field. The Schrödinger equation (4) contains terms with factors $\frac{1}{\mu_{x}}$ and $\frac{1}{\mu_{y}}$, and these terms have significantly different contributions to the binding energies and energy contributions from the magnetic field. To illustrate these effects, we performed calculations for the FS monolayer (encapsulated by $\mathrm{hBN}$ ) for mass set 1 by turning on/off kinetic and magnetic terms in (4) to evaluate the binding energies and energy contributions from the magnetic field. The results of these calculations are presented in Fig. 2(b). Omitting the kinetic term with the factor $\frac{1}{\mu_{x}}$, we obtain a binding energy of $1654.3(866.9) \mathrm{meV}$; omitting the term containing $\frac{1}{\mu_{y}}$ leads to 972.9 (360.6) $\mathrm{meV}$. Due to mass anisotropy, the kinetic energy is larger for the term related to the $x$-direction, where the reduced mass $\mu_{x}$ is lower. Therefore, the term proportional to $1 / \mu_{y}$ dominates in the exciton binding energy. The energy contribution at $B=60 \mathrm{~T}$ (omitting the term with the factor $\frac{1}{\mu_{x}}$ ) is $0.010(0.005) \mathrm{meV}$; omitting the terms with the factor $\frac{1}{\mu_{y}}$ leads to $0.97(1.70) \mathrm{meV}$.

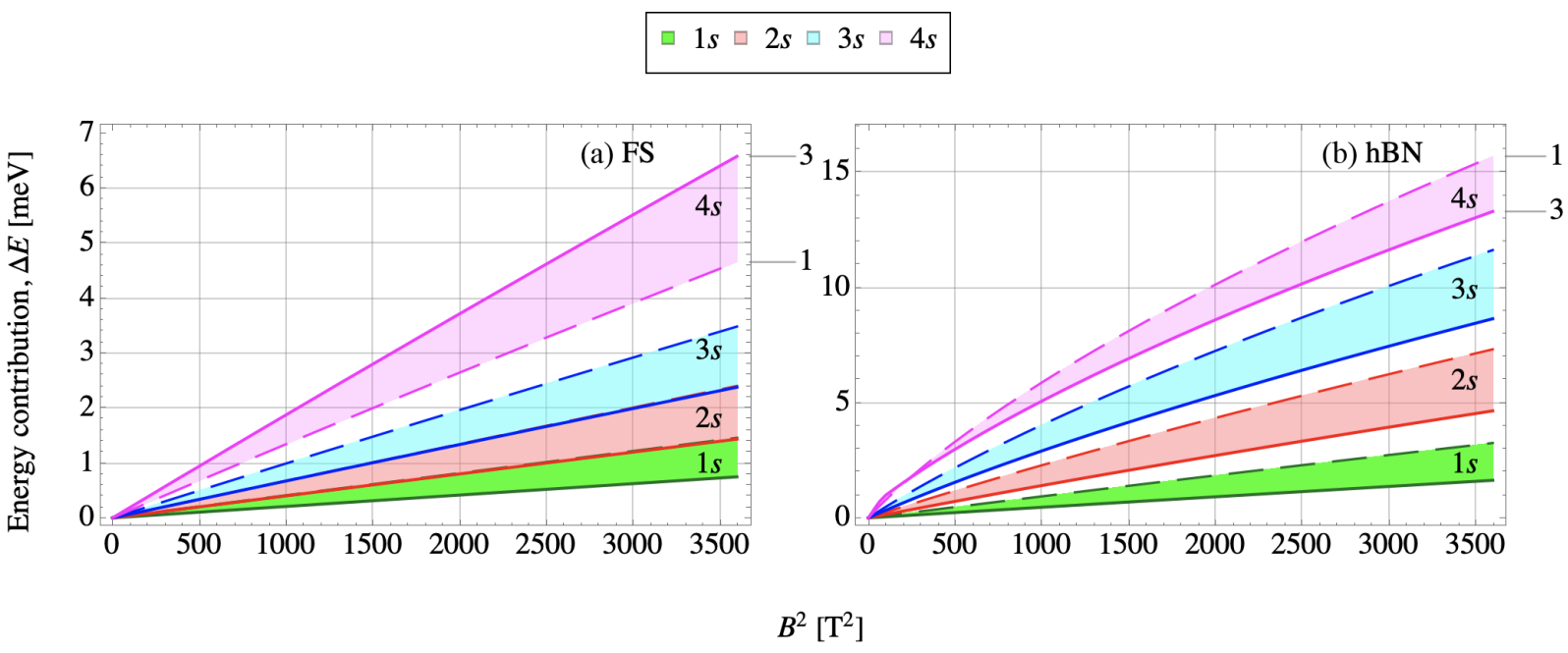

FIG. 3. Dependencies of the energy contribution of the magnetic field to the binding energies of magnetoexcitons in states $1 s, 2 s, 3 s$, and $4 s$ for (a) FS and (b) hBN-encapsulated phosphorene monolayers on the square of the magnetic field. The boundary dashed and solid curves correspond to the sets of masses 1 and 3, respectively. The contributions for the sets of masses 2 and 4 fall within the shaded region. 
Therefore, we observe that terms with $\mu_{x}$ yield higher energy contributions from the magnetic field to the binding energy. This is consistent with the anisotropic behavior of the potential $W(x, y)$, shown in Fig. 2(a), which exhibits anisotropy with respect to the armchair and zigzag directions. In summary, due to mass anisotropy, the term proportional to $1 / \mu_{y}$ dominates in $E_{B}$, while the term proportional to $1 / \mu_{x}$ dominates in $\Delta E$. It is worth mentioning that $E_{B} \gg \Delta E$. Thus, the binding energy of magnetoexcitons is strongly correlated with the reduced mass anisotropy that makes magnetoexcitons in phosphorene effectively quasi-1D quasiparticles due to the dominant presence of heavy $\mu_{y}$ effective reduced mass.

Therefore, it should be noted that, in contrast to the binding energies of direct excitons in TMDCs and Xenes, the binding energies of direct excitons in phosphorene have a more complicated dependence on the anisotropic reduced masses. In phosphorene, the central symmetry is broken considerably due to the large anisotropy of the electron and hole effective masses. Larger values of $\frac{\mu_{y}}{\mu_{x}}$ give rise to more anisotropy, causing the system to become quasi-one-dimensional [75]. The spatial distribution of the wave functions of these magnetoexcitons is strongly anisotropic. These magnetoexcitons form stripelike patterns, similar to those in nanotubes [83] or nanowires [84], that are 1D systems.

Interestingly, set 1 (with the least $\mu_{x}$ and the greatest $\mu_{y}$ ) has the lowest $1 s$ state $E_{B}$; set 3 (with the greatest $\mu_{x}$ and the least $\mu_{y}$ ) has the lowest $2 s, 3 s$, and $4 s$ binding energies. The binding-energy percent difference between sets 1 and 3 in states $2 s, 3 s$, and $4 s$ is $6 \%, 7 \%$, and $11 \%$, respectively. Also, based on Fig. 3(a), for states $1 s-3 s$, sets 1 and 3 give the highest and the lowest $\Delta E$, respectively. However, one can note that for the state $4 s$, set 3 gives the highest $\Delta E$, and set 1 gives the lowest $\Delta E$. This kind of behavior is also observed for sets 2 and 4 for states $3 s$ and $4 s$. This is not an artifact, but rather the result of the input mass parameters. The latter leads to the natural conclusion that input parameters affect the behavior of wave functions of the system. In contrast to magnetoexcitons, which dissociate in TMDC and Xene monolayers hBN-encapsulated [15], the magnetoexcitons in phosphorene monolayers in states $3 s$ and $4 s$ remain bound when the magnetic field is varied from 0 to $60 \mathrm{~T}$. This feature is also the result of the quasi-1D character of magnetoexcitons in phosphorene. The anisotropic nature of electron and hole masses leads to the domination of heavy $\mu_{y}$ that is a few times larger than the reduced mass of excitons in TMDCs, resulting in more tightly bound $s$ states.

\section{B. Contribution of the external magnetic field to the binding energies of magnetoexcitons in bilayer and vdW heterostructures}

For indirect magnetoexcitons in the FS bilayer phosphorene and the vdW heterostructure, we examine how interaction potential affects Rydberg state binding energies and the energy contribution of the external magnetic field to the binding energies. In addition, for the vdW heterostructure, we consider tuning the binding energies by the number of hBN layers.

In Fig. 4, we report results for FS bilayer for sets 1 and 3 , which give an upper and lower bound on $\Delta E$, respectively.
TABLE III. Binding energies for indirect excitons in the van der Waals heterostructures calculated using $V_{\mathrm{RK}}$ and $V_{C}$ potentials for the set of masses 3. Energies are given in $\mathrm{meV}$.

\begin{tabular}{lccccccccc}
\hline \hline & \multicolumn{4}{c}{$V_{\mathrm{RK}}$} & & \multicolumn{5}{c}{$V_{C}$} \\
\cline { 2 - 7 } \cline { 8 - 10 }$N$ & $1 s$ & $2 s$ & $3 s$ & $4 s$ & & $1 s$ & $2 s$ & $3 s$ & $4 s$ \\
\hline 1 & 98.50 & 55.13 & 37.45 & 28.11 & & 128.38 & 66.00 & 43.02 & 30.91 \\
2 & 86.39 & 51.29 & 35.27 & 27.15 & & 104.90 & 58.98 & 39.30 & 29.39 \\
3 & 76.91 & 47.76 & 33.31 & 26.15 & & 89.28 & 53.38 & 36.34 & 27.96 \\
4 & 69.32 & 44.60 & 31.55 & 25.16 & & 78.03 & 48.84 & 33.90 & 26.64 \\
5 & 60.10 & 41.80 & 29.96 & 24.30 & & 69.49 & 45.06 & 31.82 & 25.43 \\
6 & 57.93 & 39.31 & 28.53 & 23.29 & & 62.75 & 41.88 & 30.03 & 24.32 \\
\hline \hline
\end{tabular}

As was observed in the monolayer, there is a reversal in lower and upper bounds in state $4 s$ in the bilayer: set 1 gives a lower bound and set 3 gives an upper bound. Similar to the results reported for magnetoexcitons in Xene and TMDC bilayer systems in Refs. [11,15], in bilayer phosphorene the following relation always holds: $\Delta E_{\mathrm{RK}}>\Delta E_{C}$. In addition, it is worth mentioning that $\Delta E$ is higher for indirect magnetoexcitons in the FS bilayer system than for direct magnetoexcitons in FS phosphorene. Based on the results in Table II for the bilayer phosphorene, Rydberg state binding energies calculated with $V_{C}$ are twice as large as the binding energies calculated with the $V_{\mathrm{RK}}$ potential. Similar to the monolayer system, magnetoexcitons in the bilayer system in states $2 s, 3 s$, and $4 s$ are more tightly bound than magnetoexcitons in the TMDC bilayer system. In Fig. 5, we compare $\Delta E_{\mathrm{RK}}$ and $\Delta E_{C}$ for the $\mathrm{vdW}$ heterostructure. Results are given for set 3 , which is taken to be the representative case. We plot $\Delta E$ as a function of the external magnetic field and the number of $\mathrm{hBN}$ layers. As can be seen from Fig. 5, as the number of hBN layers increases, $\Delta E_{\mathrm{RK}}$ and $\Delta E_{C}$ converge. Once again, in contrast to indirect magnetoexcitons in TMDCs and Xenes doublelayer heterostructures, magnetoexcitons in vdW phosphorene heterostructures remain bound in states $3 s$ and $4 s$ while the magnetic field is varied between 0 and $60 \mathrm{~T}$. This can be explained by the fact that the binding energies of states $3 s$ and $4 s$ are higher for indirect magnetoexcitons in phosphorene due to their effectively quasi-one-dimensional nature, which is due in large part to the dominance of heavy $\mu_{y}$. The quasi-onedimensionality of the magnetoexcitons in phosphorene is also demonstrated by the comparison of $\Delta E$ of the vdW phosphorene heterostructure shown in Fig. 5 and that of the TMDC heterostructures: $\Delta E$ due to the magnetic field in the $\mathrm{vdW}$ phosphorene heterostructure is always higher than that of the TMDC heterostructures if we consider only the term with the factor $\frac{1}{\mu_{x}}$. In Table III, we report the binding energies of Rydberg states for $N=1,2,3,4,5,6$ when Eq. (4) is solved with $V_{\mathrm{RK}}$ and $V_{C}$. The binding energies calculated using the Coulomb potential are always larger than those obtained with the RK potential. The increase of the phosphorene interlayer distance in the vdW heterostructure leads to the deduction of electron-hole interaction and results in the decrease in $E_{B}$ of indirect magnetoexcitons. 


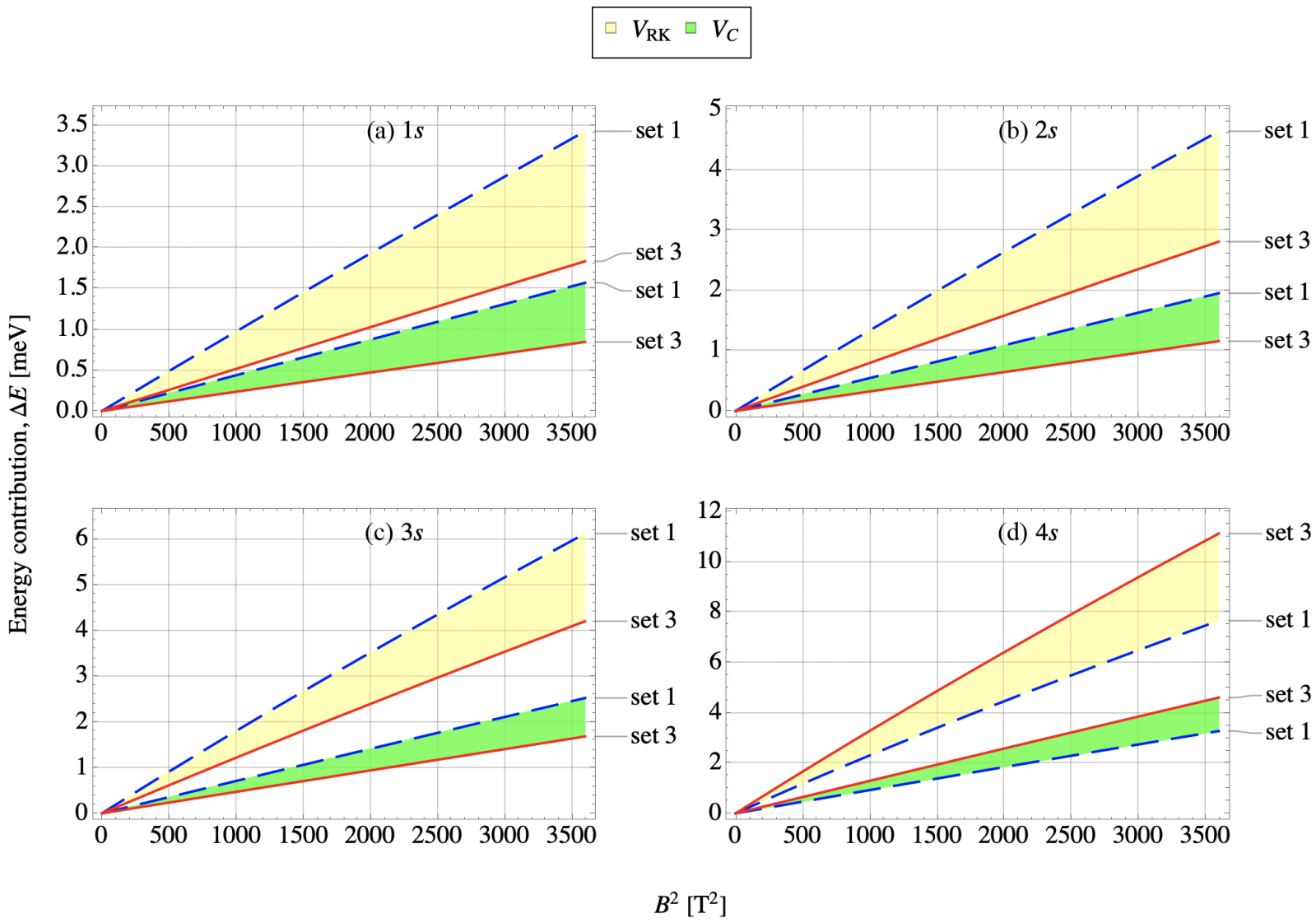

FIG. 4. The energy contribution of the magnetic field to the binding energy of Rydberg states $1 s-4 s$ for the FS bilayer obtained using $V_{\mathrm{RK}}$ and $V_{C}$ potentials. The boundary dashed and solid curves correspond to the sets of masses 1 and 3, respectively. The yellow and green shaded areas correspond to the calculations with $V_{\mathrm{RK}}$ and $V_{C}$ potentials, respectively.

\section{DIAMAGNETIC COEFFICIENTS}

In this paper, we calculate the diamagnetic coefficients in the same fashion as in Refs. [11,15,59]. The diamagnetic coefficients for magnetoexcitons in Rydberg states in phosphorene are reported. The magnetic field range of 0-30 T has been used to calculated the DMCs. In this magnetic field range, one can observe the linear dependence of energy on $B^{2}$. Here, we follow the notation in the literature and denote the diamagnetic coefficient as $\sigma$. We report the diamagnetic coefficients for magnetoexcitons in monolayer and bilayer systems, as well as in vdW heterostructures, in TablesV and IV, respectively, for two sets of masses. $\sigma$ for the FS and hBN-encapsulated phosphorene monolayers is obtained when the magnetoexcitons interact through the $V_{\mathrm{RK}}$ interaction; for the bilayer, $\sigma$ is given when Eq. (4) is solved using both the $V_{\mathrm{RK}}$ and $V_{C}$ potentials. In the monolayer, $\sigma_{1}>\sigma_{3}$, where $\sigma_{1}$ and $\sigma_{3}$ are DMCs obtained with sets of masses 1 and 3, respectively. For phosphorene encapsulated by hBN, $\sigma$ can only be extracted in states $1 s$, $2 s$, and $3 s$. In the case of FS phosphorene, $\sigma$ can also be extracted in the state $4 s$. In the case of bilayer phosphorene, the diamagnetic coefficients can be extracted for the $1 s, 2 s$, $3 s$, and $4 s$ states, unlike the diamagnetic coefficients of the bilayer system composed of TMDCs [11], for which $\sigma$ can be extracted for all examined states.
The DMCs for the vdW heterostructure are obtained using the $V_{\mathrm{RK}}$ and $V_{C}$ potentials. $\sigma$ can be extracted for all mass sets in the state $1 s$ and for the mass set 3 in the state $2 s$ for all values of examined $N$.

\section{CONCLUSIONS}

Within the framework of the Mott-Wannier model, we study direct and indirect excitons in Rydberg states in phosphorene monolayers, bilayer, and $\mathrm{vdW}$ heterostructure in

TABLE IV. The diamagnetic coefficients, $\sigma$, of direct magnetoexcitons in FS and hBN-encapsulated monolayers for sets of masses 1 and 3. $\sigma$ is given in $\mu \mathrm{eV} / \mathrm{T}^{2}$. DMCs are obtained for the magnetic field range of 0-30 T, with $R^{2}=0.9998$ for the linear regression model.

\begin{tabular}{|c|c|c|c|c|c|c|c|c|}
\hline \multirow[b]{3}{*}{ State } & \multicolumn{4}{|c|}{ Monolayer } & \multicolumn{4}{|c|}{ Bilayer } \\
\hline & \multicolumn{2}{|c|}{ Set 1} & \multicolumn{2}{|c|}{ Set 3} & \multicolumn{2}{|c|}{ Set 1} & \multicolumn{2}{|c|}{ Set 3} \\
\hline & $\mathrm{hBN}$ & FS & $\mathrm{hBN}$ & FS & $V_{\mathrm{RK}}$ & $V_{C}$ & $V_{\mathrm{RK}}$ & $V_{C}$ \\
\hline $1 s$ & 0.94 & 0.41 & 0.46 & 0.21 & 0.97 & 0.44 & 0.52 & 0.23 \\
\hline $2 s$ & 2.31 & 0.68 & 1.41 & 0.40 & 1.34 & 0.55 & 0.80 & 0.32 \\
\hline $3 s$ & 4.13 & 0.99 & 2.96 & 0.67 & 1.81 & 0.71 & 1.22 & 0.47 \\
\hline $4 s$ & & 1.34 & & 1.88 & 2.32 & 0.93 & 3.30 & 1.30 \\
\hline
\end{tabular}


(a) $1 \mathrm{~s}$

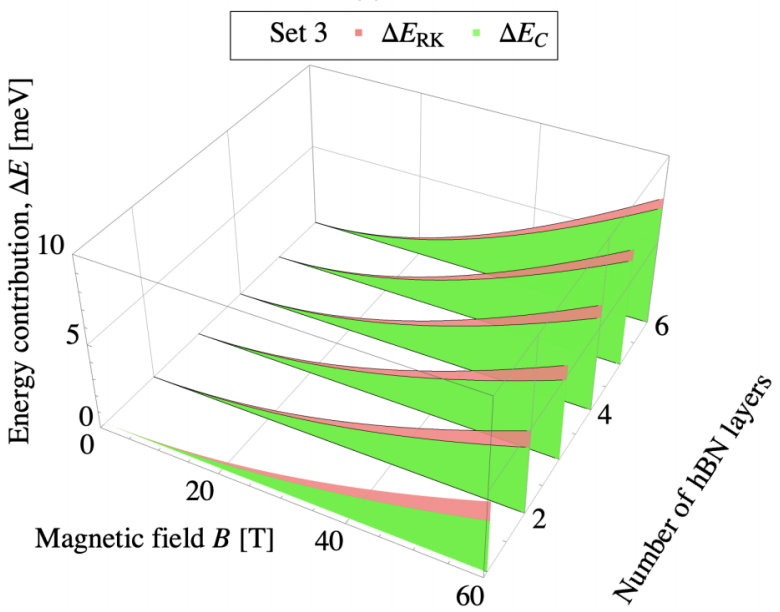

(c) $3 \mathrm{~s}$

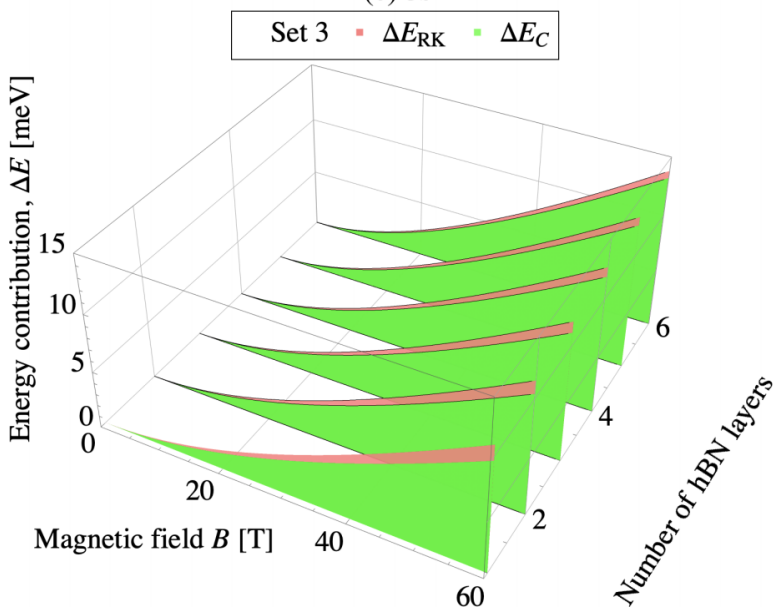

(b) $2 \mathrm{~s}$

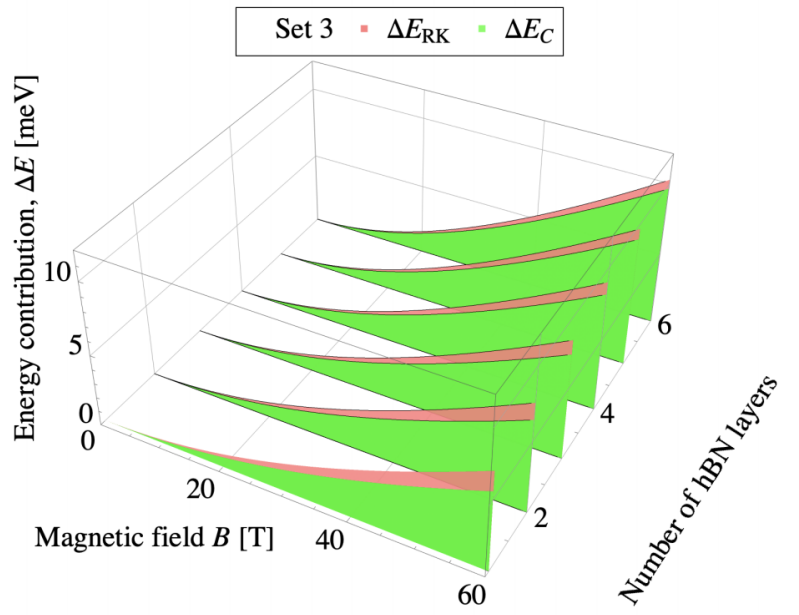

(d) $4 \mathrm{~s}$

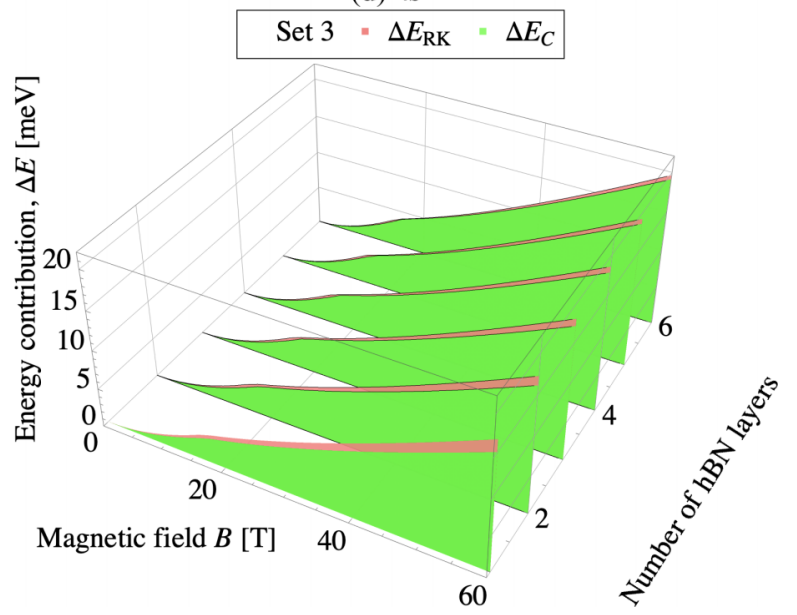

FIG. 5. The energy contribution for indirect magnetoexcitons in vdW heterostructures as a function of the magnetic field and the number of hBN layers. The data are plotted for the set of masses 3 .

TABLE V. The diamagnetic coefficients, $\sigma$, of indirect magnetoexcitons in the vdW heterostructure for the sets of masses 1 and 3. $\sigma$ is given in $\mu \mathrm{eV} / \mathrm{T}^{2}$. DMCs are obtained for the range of the magnetic field between 0 and $30 \mathrm{~T}$ and correspond to $R^{2}=0.9998$ for the linear regression model.

\begin{tabular}{|c|c|c|c|c|c|}
\hline \multirow[b]{2}{*}{ State } & \multirow[b]{2}{*}{$N$} & \multicolumn{2}{|c|}{ Set 1} & \multicolumn{2}{|c|}{ Set 3} \\
\hline & & $V_{\mathrm{RK}}$ & $V_{C}$ & $V_{\mathrm{RK}}$ & $V_{C}$ \\
\hline \multirow{6}{*}{$1 s$} & 1 & 2.36 & 1.72 & 1.24 & 0.88 \\
\hline & 2 & 2.80 & 2.22 & 1.49 & 1.16 \\
\hline & 3 & 3.23 & 2.72 & 1.74 & 1.45 \\
\hline & 4 & 3.67 & 3.20 & 2.00 & 1.73 \\
\hline & 5 & 4.09 & 3.68 & 2.27 & 2.02 \\
\hline & 6 & 4.51 & 4.14 & 2.53 & 2.30 \\
\hline \multirow{6}{*}{$2 s$} & 1 & 3.64 & 2.85 & 2.24 & 1.73 \\
\hline & 2 & 4.04 & 3.37 & 2.50 & 2.06 \\
\hline & 3 & 4.46 & 3.88 & 2.77 & 2.39 \\
\hline & 4 & 4.88 & 4.38 & 3.05 & 2.71 \\
\hline & 5 & & & 3.34 & 3.03 \\
\hline & 6 & & & 3.62 & 3.35 \\
\hline $3 s$ & 1 & & & & 3.39 \\
\hline
\end{tabular}

the presence of an external magnetic field applied perpendicular to the monolayer or heterostructure. We calculate the binding energies and DMCs for the Rydberg states $1 s, 2 s, 3 s$, and $4 s$ for magnetoexcitons formed in these systems.

The anisotropy of the effective masses of electrons and holes heavily influences both the binding energy of excitons and the magnetic field contribution to the binding energy of magnetoexcitons in phosphorene. Phosphorene anisotropy manifests as follows: higher $\mu_{y}$ leads to sizable $E_{B}$ but to very little energy contribution of the magnetic field, whereas smaller $\mu_{x}$ gives a little contribution to $E_{B}$ but a sizable contribution to $\Delta E$. Therefore, the binding energies of magnetoexcitons are strongly correlated with the reduced mass anisotropy, which, due to the predominance of heavy $\mu_{y}$, renders magnetoexcitons in phosphorene as effectively quasi-1D quasiparticles.

The DMCs demonstrate the strong dependence on the effective electron-hole masses: DMCs in phosphorene monolayers, bilayers, and vdW heterostructures for set 1 are about twice as large as those for set 3 . The other distinct feature of the diamagnetic coefficients of the bilayer and vdW heterostructures is that $\sigma$, when calculated using the Coulomb 
potential, is always smaller than DMCs obtained using the Rytova-Keldysh potential. In other words, the screening increases the diamagnetic coefficients.

Our calculations show strong sensitivity of the binding energies and DMCs of direct and indirect magnetoexcitons on the values of the effective electron and hole masses. However, there is a discrepancy between mass values obtained using different methods [26,68,75-77,85-90]. Therefore, it is important to have reliable values of the effective electron and hole masses.

We show that the binding energy of direct and indirect magnetoexcitons can be tuned by the external magnetic field. Furthermore, $E_{B}$ and DMC in vdW heterostructures could be tuned efficiently by the stacking of hBN layers. Such tunability of binding energies and DMCs is potentially useful for the design of devices.
Finally, in summary, we have shown that the vdW phosphorene heterostructure is a novel category of 2D semiconductors that offers a tunability of magnetoexciton binding energies by means of an external magnetic field and by control of the number of $\mathrm{hBN}$ layers separating two phosphorene sheets. Thus, phosphorene provides a unique platform for novel optoelectronic applications and the exploration of the role of symmetry breaking in anisotropic exciton physics.

\section{ACKNOWLEDGMENTS}

This work is supported by the U.S. Department of Defense under Grant No. W911NF1810433 and PSC-CUNY Award No. 62261-00 50.
[1] A. H. Castro Neto, F. Guinea, N. M. R. Peres, K. S. Novoselov, and A. K. Geim, The electronic properties of graphene, Rev. Mod. Phys. 81, 109 (2009).

[2] P. Avouris, T. F. Heinz, and T. Low, 2D Materials: Properties and Devices (Cambridge University Press, Cambridge, 2017).

[3] R. Cheng, D. Li, H. Zhou, C. Wang, A. Yin, S. Jiang, Y. Liu, Y. Chen, Y. Huang, and X. Duan, Electroluminescence and photocurrent generation from atomically sharp $\mathrm{WSe}_{2} / \mathrm{MoS}_{2}$ heterojunction p-n diodes, Nano Lett. 14, 5590 (2014).

[4] C.-H. Lee, G.-H. Lee, A. Zande, W. Chen, Y. Li, M. Han, X. Cui, G. Arefe, C. Nuckolls, T. Heinz et al., Atomically thin p-n junctions with van der Waals heterointerfaces, Nat. Nanotechnol. 9, 676 (2014).

[5] O. Cotleţ, S. Zeytinoğlu, M. Sigrist, E. Demler, and A. Imamoğlu, Superconductivity and other collective phenomena in a hybrid Bose-Fermi mixture formed by a polariton condensate and an electron system in two dimensions, Phys. Rev. B 93, 054510 (2016).

[6] S. Manzeli, D. Ovchinnikov, D. Pasquier, O. V. Yazyev, and A. Kis, 2D transition metal dichalcogenides, Nat. Rev. Mater. 2, 17033 (2017).

[7] O. L. Berman and R. Ya. Kezerashvili, High-temperature superfluidity of the two-component Bose gas in a transition metal dichalcogenide bilayer, Phys. Rev. B 93, 245410 (2016).

[8] O. L. Berman and R. Ya. Kezerashvili, Superfluidity of dipolar excitons in a transition metal dichalcogenide double layer, Phys. Rev. B 96, 094502 (2017).

[9] G. Wang, A. Chernikov, M. M. Glazov, T. F. Heinz, X. Marie, T. Amand, and B. Urbaszek, Colloquium: Excitons in atomically thin transition metal dichalcogenides, Rev. Mod. Phys. 90, 021001 (2018).

[10] M. N. Brunetti, O. L. Berman, and R. Ya. Kezerashvili, Optical absorption by indirect excitons in a transition metal dichalcogenide/hexagonal boron nitride heterostructure, J. Phys.: Condens. Matter 30, 225001 (2018).

[11] R. Ya. Kezerashvili and A. Spiridonova, Magnetoexcitons in transition metal dichalcogenide monolayers, bilayers, and van der Waals heterostructures, Phys. Rev. Research 3, 033078 (2021).
[12] L. Matthes, O. Pulci, and F. Bechstedt, Massive dirac quasiparticles in the optical absorbance of graphene, silicene, germanene, and tinene, J. Phys.: Condens. Matter 25, 395305 (2013).

[13] A. Molle, J. Goldberger, M. Houssa, Y. Xu, S.-C. Zhang, and D. Akinwande, Buckled two-dimensional Xene sheets, Nat. Mater 16, 163 (2017).

[14] J. Zheng, Y. Xiang, C. Li, R. Yuan, F. Chi, and Y. Guo, AllOptically Controlled Topological Transistor Based on Xenes, Phys. Rev. Appl. 14, 034027 (2020).

[15] R. Ya. Kezerashvili and A. Spiridonova, Effects of parallel electric and magnetic fields on Rydberg excitons in buckled two-dimensional materials, Phys. Rev. B 103, 165410 (2021).

[16] L. Li, Y. Yu, G. J. Ye, Q. Ge, X. Ou, H. Wu, D. Feng, X. H. Chen, and Y. Zhang, Black phosphorus field-effect transistors, Nat. Nanotechnol. 9, 372 (2014).

[17] H. Liu, A. T. Neal, Z. Zhu, Z. Luo, X. Xu, D. Tománek, and P. D. Ye, Phosphorene: An unexplored 2D semiconductor with a high hole mobility, ACS Nano 8, 4033 (2014).

[18] K. F. Mak, C. Lee, J. Hone, J. Shan, and T. F. Heinz, Atomically thin $\mathrm{MoS}_{2}$ : A New Direct-Gap Semiconductor, Phys. Rev. Lett. 105, 136805 (2010).

[19] A. Splendiani, L. Sun, Y. Zhang, T. Li, J. Kim, C.-Y. Chim, G. Galli, and F. Wang, Emerging photoluminescence in monolayer $\mathrm{MoS}_{2}$, Nano Lett. 10, 1271 (2010).

[20] S. Tongay, J. Zhou, C. Ataca, K. Lo, T. S. Matthews, J. Li, J. C. Grossman, and J. Wu, Thermally driven crossover from indirect toward direct bandgap in 2D semiconductors: $\mathrm{MoSe}_{2}$ versus $\mathrm{MoS}_{2}$, Nano Lett. 12, 5576 (2012).

[21] P. W. Bridgman, Two new modifications of phosphorus, J. Am. Chem. Soc. 36, 1344 (1914).

[22] A. Carvalho, M. Wang, X. Zhu, A. S. Rodin, H. Su, and A. H. Castro Neto, Phosphorene: From theory to applications, Nat. Rev. Mater. 1, 16061 (2016).

[23] M. Batmunkh, M. Bat-Erdene, and J. G. Shapter, Phosphorene and phosphorene-based materials-prospects for future applications, Adv. Mater. 28, 8586 (2016).

[24] D. Y. Qiu, F. H. da Jornada, and S. G. Louie, Environmental screening effects in 2D materials: Renormalization of the bandgap, electronic structure, and optical spectra of few-layer black phosphorus, Nano Lett. 17, 4706 (2017). 
[25] Y. Takao and A. Morita, Electronic structure of black phosphorus: Tight binding approach, Physica B+C 105, 93 (1981).

[26] T. Low, A. S. Rodin, A. Carvalho, Y. Jiang, H. Wang, F. Xia, and A. H. Castro Neto, Tunable optical properties of multilayer black phosphorus thin films, Phys. Rev. B 90, 075434 (2014).

[27] M. Ezawa, Topological origin of quasi-flat edge band in phosphorene, New J. Phys. 16, 115004 (2014).

[28] M. N. Brunetti, O. L. Berman, and R. Ya. Kezerashvili, Optical properties of anisotropic excitons in phosphorene, Phys. Rev. B 100, 155433 (2019).

[29] S. Yoon, T. Kim, S.-Y. Seo, S.-H. Shin, S.-B. Song, B. J. Kim, K. Watanabe, T. Taniguchi, G.-H. Lee, M.-H. Jo et al., Electrical control of anisotropic and tightly bound excitons in bilayer phosphorene, Phys. Rev. B 103, L041407 (2021).

[30] S. P. Koenig, R. A. Doganov, H. Schmidt, A. H. Castro Neto, and B. Özyilmaz, Electric field effect in ultrathin black phosphorus, Appl. Phys. Lett. 104, 103106 (2014).

[31] A. Chaves, T. Low, P. Avouris, D. Çakır, and F. M. Peeters, Anisotropic exciton Stark shift in black phosphorus, Phys. Rev. B 91, 155311 (2015).

[32] P. Le and M. Yarmohammadi, Tuning thermoelectric transport in phosphorene through a perpendicular magnetic field, Chem. Phys. 519, 1 (2019).

[33] H. Kamban and T. Pedersen, Interlayer excitons in van der Waals heterostructures: Binding energy, Stark shift, and fieldinduced dissociation, Sci. Rep. 10, 5537 (2020).

[34] A. S. Rodin, A. Carvalho, and A. H. Castro Neto, StrainInduced Gap Modification in Black Phosphorus, Phys. Rev. Lett. 112, 176801 (2014).

[35] Y. Li, S. Yang, and J. Li, Modulation of the electronic properties of ultrathin black phosphorus by strain and electrical field, J. Phys. Chem. C 118, 23970 (2014).

[36] H. Y. Lv, W. J. Lu, D. F. Shao, and Y. P. Sun, Enhanced thermoelectric performance of phosphorene by strain-induced band convergence, Phys. Rev. B 90, 085433 (2014).

[37] X. Zhou, W.-K. Lou, F. Zhai, and K. Chang, Anomalous magneto-optical response of black phosphorus thin films, Phys. Rev. B 92, 165405 (2015).

[38] X. Y. Zhou, R. Zhang, J. P. Sun, Y. L. Zou, D. Zhang, W. K. Lou, F. Cheng, G. H. Zhou, F. Zhai, and K. Chang, Landau levels and magneto-transport property of monolayer phosphorene, Sci. Rep. 5, 12295 (2015).

[39] M. Tahir, P. Vasilopoulos, and F. M. Peeters, Magneto-optical transport properties of monolayer phosphorene, Phys. Rev. B 92, 045420 (2015).

[40] P. E. Faria Junior, M. Kurpas, M. Gmitra, and J. Fabian, $k \cdot p$ theory for phosphorene: Effective $g$-factors, Landau levels, and excitons, Phys. Rev. B 100, 115203 (2019).

[41] J. Zhou, T.-Y. Cai, and S. Ju, Anisotropic exciton excitations and optical properties of hittorf's phosphorene, Phys. Rev. Research 2, 033288 (2020).

[42] J.-Y. Wu, W.-P. Su, and G. Gumbs, Anomalous magnetotransport properties of bilayer phosphorene, Sci. Rep. 10, 7674 (2020).

[43] V. Tran, R. Fei, and L. Yang, Quasiparticle energies, excitons, and optical spectra of few-layer black phosphorus, 2D Mater. 2, 044014 (2015).

[44] X. Wang, A. M. Jones, K. L. Seyler, V. Tran, Y. Jia, H. Zhao, H. Wang, L. Yang, X. Xu, and F. Xia, Highly anisotropic and robust excitons in monolayer black phosphorus, Nat. Nanotechnol. 10, 517 (2015).

[45] R. Tian, R. Fei, S. Hu, T. Li, B. Zheng, Y. Shi, J. Zhao, L. Zhang, X. Gan, and X. Wang, Observation of excitonic series in monolayer and few-layer black phosphorus, Phys. Rev. B 101, 235407 (2020).

[46] J. C. G. Henriques and N. M. R. Peres, Excitons in phosphorene: A semi-analytical perturbative approach, Phys. Rev. B 101, 035406 (2020).

[47] R. Elliott and R. Loudon, Theory of the absorption edge in semiconductors in a high magnetic field, J. Phys. Chem. Solids 15, 196 (1960).

[48] H. Hasegawa and R. Howard, Optical absorption spectrum of hydrogenic atoms in a strong magnetic field, J. Phys. Chem. Solids 21, 179 (1961).

[49] M. Shinada and S. Sugano, Optical absorption edge in layertype semiconductors, J. Phys. Soc. Jpn. 20, 1274 (1965).

[50] L. P. Gorkov and I. E. Dzualoshinskii, Contribution to the theory of the Mott exciton in a strong magnetic field, Zh. Eksp. Teor. Fiz. 53, 717 (1967).

[51] O. Akimoto and H. Hasegawa, Interband optical transitions in extremely anisotropic semiconductors. II. Coexistence of exciton and the Landau levels, J. Phys. Soc. Jpn. 22, 181 (1967).

[52] S. N. Walck and T. L. Reinecke, Exciton diamagnetic shift in semiconductor nanostructures, Phys. Rev. B 57, 9088 (1998).

[53] F. Luckert, M. Yakushev, C. Faugeras, A. Karotki, A. Mudryi, and R. Martin, Diamagnetic shift of the A free exciton in $\mathrm{CuGaSe}_{2}$ single crystals, Appl. Phys. Lett. 97, 162101 (2010).

[54] B. Choi, Y. Kim, and J. Song, Diamagnetic shift of a InGaP-AlInGaP semiconductor single quantum well under pulsed-magnetic fields, Appl. Sci. Converg. Technol. 24, 156 (2015).

[55] M. Van der Donck, M. Zarenia, and F. M. Peeters, Excitons, trions, and biexcitons in transition-metal dichalcogenides: Magnetic-field dependence, Phys. Rev. B 97, 195408 (2018).

[56] M. Van der Donck, M. Zarenia, and F. M. Peeters, Strong valley Zeeman effect of dark excitons in monolayer transition metal dichalcogenides in a tilted magnetic field, Phys. Rev. B 97, 081109(R) (2018).

[57] A. V. Stier, N. P. Wilson, K. A. Velizhanin, J. Kono, X. Xu, and S. A. Crooker, Magnetooptics of Exciton Rydberg States in a Monolayer Semiconductor, Phys. Rev. Lett. 120, 057405 (2018).

[58] B. Han, C. Robert, E. Courtade, M. Manca, S. Shree, T. Amand, P. Renucci, T. Taniguchi, K. Watanabe, X. Marie, L. E. Golub, M. M. Glazov, and B. Urbaszek, Exciton States in Monolayer $\mathrm{MoSe}_{2}$ and $\mathrm{MoTe}_{2}$ probed by upconversion spectroscopy, Phys. Rev. X 8, 031073 (2018).

[59] A. Spiridonova, Magnetoexcitons in monolayer transitionmetal dichalcogenides, Phys. Lett. A 384, 126850 (2020).

[60] Y. Li, J. Ludwig, T. Low, A. Chernikov, X. Cui, G. Arefe, Y. D. Kim, A. M. van der Zande, A. Rigosi, H. M. Hill, S. H. Kim, J. Hone, Z. Li, D. Smirnov, and T. F. Heinz, Valley Splitting and Polarization by the Zeeman Effect in Monolayer $\mathrm{MoSe}_{2}$, Phys. Rev. Lett. 113, 266804 (2014).

[61] A. Srivastava, M. Sidler, A. Allain, D. Lembke, A. Kis, and A. Imamoglu, Valley Zeeman effect in elementary optical excitations of a monolayer $\mathrm{WSe}_{2}$, Nat. Phys. 11, 141 (2014).

[62] G. Aivazian, Z. Gong, A. Jones, R.-L. Chu, J.-Q. Yan, D. Mandrus, C. Zhang, D. Cobden, W. Yao, and X. Xu, Magnetic 
control of valley pseudospin in monolayer $\mathrm{WSe}_{2}$, Nat. Phys. 11, 148 (2015).

[63] D. MacNeill, C. Heikes, K. F. Mak, Z. Anderson, A. Kormányos, V. Zólyomi, J. Park, and D. C. Ralph, Breaking of Valley Degeneracy by Magnetic Field in Monolayer $\mathrm{MoSe}_{2}$, Phys. Rev. Lett. 114, 037401 (2015).

[64] G. Plechinger, P. Nagler, A. Arora, A. Aguila, M. Ballottin, T. Frank, P. Steinleitner, M. Gmitra, J. Fabian, P. Christianen et al., Excitonic valley effects in monolayer $\mathrm{WS}_{2}$ under high magnetic fields, Nano Lett. 16, 7899 (2016).

[65] A. Stier, K. Mccreary, B. Jonker, J. Kono, and S. Crooker, Exciton diamagnetic shifts and valley Zeeman effects in monolayer $\mathrm{WS}_{2}$ and $\mathrm{MoS}_{2}$ to 65 Tesla, Nat. Commun. 7, 10643 (2016).

[66] N. Rytova, Screened potential of a point charge in a thin film, Proc. Moscow State University, Phys. Astron. 3, 30 (1967).

[67] L. V. Keldysh, Coulomb interaction in thin semiconductor and semimetal films, JETP Lett. 29, 658 (1979).

[68] J. Qiao, X. Kong, Z.-X. Hu, F. Yang, and W. Ji, High-mobility transport anisotropy and linear dichroism in few-layer black phosphorus, Nat. Commun. 5, 4475 (2014).

[69] A. Ziletti, A. Carvalho, D. K. Campbell, D. F. Coker, and A. H. Castro Neto, Oxygen Defects in Phosphorene, Phys. Rev. Lett. 114, 046801 (2015).

[70] A. Ziletti, A. Carvalho, P. E. Trevisanutto, D. K. Campbell, D. F. Coker, and A. H. Castro Neto, Phosphorene oxides: Bandgap engineering of phosphorene by oxidation, Phys. Rev. B 91, 085407 (2015).

[71] T. C. Berkelbach, M. S. Hybertsen, and D. R. Reichman, Theory of neutral and charged excitons in monolayer transition metal dichalcogenides, Phys. Rev. B 88, 045318 (2013).

[72] L. D. Landau and E. M. Lifshitz, Quantum Mechanics: Non-relativistic Theory, 3rd ed., revised and enlarged edition (Pergamon, Oxford, 1977).

[73] A. H. MacDonald and D. S. Ritchie, Hydrogenic energy levels in two dimensions at arbitrary magnetic fields, Phys. Rev. B 33, 8336 (1986).

[74] Y. Lozovik and A. Ruvinsky, Magnetoexcitons in coupled quantum wells, Phys. Lett. A 227, 271 (1997).

[75] X. Peng, Q. Wei, and A. Copple, Strain-engineered directindirect band gap transition and its mechanism in twodimensional phosphorene, Phys. Rev. B 90, 085402 (2014).

[76] V. Tran and L. Yang, Scaling laws for the band gap and optical response of phosphorene nanoribbons, Phys. Rev. B 89, 245407 (2014).

[77] C. J. Páez, K. DeLello, D. Le, A. L. C. Pereira, and E. R. Mucciolo, Disorder effect on the anisotropic resistivity of phosphorene determined by a tight-binding model, Phys. Rev. B 94, 165419 (2016).
[78] A. S. Rodin, A. Carvalho, and A. H. Castro Neto, Excitons in anisotropic two-dimensional semiconducting crystals, Phys. Rev. B 90, 075429 (2014).

[79] P. Kumar, B. S. Bhadoria, S. Kumar, S. Bhowmick, Y. S Chauhan, and A. Agarwal, Thickness and electric-fielddependent polarizability and dielectric constant in phosphorene, Phys. Rev. B 93, 195428 (2016).

[80] V. Tran, R. Soklaski, Y. Liang, and L. Yang, Layer-controlled band gap and anisotropic excitons in few-layer black phosphorus, Phys. Rev. B 89, 235319 (2014).

[81] A. Castellanos-Gomez, L. Vicarelli, E. Prada, J. O. Island, K. L. Narasimha-Acharya, S. I. Blanter, D. J. Groenendijk, M. Buscema, G. A. Steele, J. V. Alvarez et al., Isolation and characterization of few-layer black phosphorus, 2D Mater. 1, 025001 (2014).

[82] H. C. Kamban, T. G. Pedersen, and N. M. R. Peres, Anisotropic Stark shift, field-induced dissociation, and electroabsorption of excitons in phosphorene, Phys. Rev. B 102, 115305 (2020).

[83] I. V. Bondarev, Asymptotic exchange coupling of quasi-onedimensional excitons in carbon nanotubes, Phys. Rev. B 83, 153409 (2011).

[84] R. Ya. Kezerashvili, Z. Machavariani, B. Beradze, and T. Tchelidze, Trions and biexcitons in $\mathrm{Zno} / \mathrm{ZnMgO}, \mathrm{CdSe} / \mathrm{ZnS}$ and $\mathrm{CdSe} / \mathrm{CdS}$ core/shell nanowires, J. Phys. E 109, 228 (2019).

[85] B. Sa, Y.-L. Li, Z. Sun, J. Qi, C. Wen, and B. Wu, The electronic origin of shear-induced direct to indirect gap transition and anisotropy diminution in phosphorene, Nanotechnology 26, 215205 (2015).

[86] Z. Jin, J. T. Mullen, and K. W. Kim, Highly anisotropic electronic transport properties of monolayer and bilayer phosphorene from first principles, Appl. Phys. Lett. 109, 053108 (2016).

[87] W. Zhang, J. Yin, P. Zhang, and Y. Ding, Strain/stress engineering on the mechanical and electronic properties of phosphorene nanosheets and nanotubes, RSC Adv. 7, 51466 (2017).

[88] F. Zhai, W. Hu, and J. Lu, Anisotropic tunneling resistance in a phosphorene-based magnetic barrier, Phys. Rev. B 96, 165416 (2017).

[89] S. Kaur, A. Kumar, S. Srivastava, K. Tankeshwar, and R. Pandey, Monolayer, bilayer, and heterostructures of green phosphorene for water splitting and photovoltaics, J. Phys. Chem. C 122, 26032 (2018).

[90] G. Gaddemane, W. G. Vandenberghe, M. L. Van de Put, S. Chen, S. Tiwari, E. Chen, and M. V. Fischetti, Theoretical studies of electronic transport in monolayer and bilayer phosphorene: A critical overview, Phys. Rev. B 98, 115416 (2018). 